\title{
Laparoscopic total gastrectomy for a giant gastrointestinal stromal tumor (GIST) with acute massive gastrointestinal bleeding: a case report
}

\author{
Mohammad Kermansaravi ${ }^{1}$, Samaneh Rokhgireh ${ }^{2}$, Sattar Darabi ${ }^{1}$, Abdolreza Pazouki ${ }^{1,3,4}$ \\ ${ }^{1}$ Minimally Invasive Surgery Research Center, Iran University of Medical Sciences, Tehran, Iran \\ 2Endometriosis and Gynecologic Disorders Research Center, Iran University of Medical Sciences, Tehran, Iran \\ ${ }^{3}$ Center of Excellence for Minimally Invasive Surgery Training, Iran University of Medical Sciences, Tehran, Iran \\ ${ }^{4}$ Center of Excellence of European Branch of International Federation for Surgery of Obesity, Tehran, Iran
}

Videosurgery Miniinv 2017; 12 (3): 306-310

DOI: https://doi.org/10.5114/wiitm.2017.67997

\begin{abstract}
Gastrointestinal stromal tumors (GISTS) include 80\% of gastrointestinal mesenchymal tumors that originate from interstitial Cajal cells and include 0.1-3\% of Gl malignancies, and the stomach is the most commonly involved organ. The only potentially curative treatment is surgical resection with clear margins. Although laparoscopic resection of small GISTs is a standard treatment, there is controversy about laparoscopic surgical resection for large and giant GISTS. A 52-year-old woman, a known case of large GIST of the stomach that was under neoadjuvant imatinib therapy, was admitted to the emergency department due to acute massive gastrointestinal bleeding (GIB). The patient underwent laparoscopic total gastrectomy and received adjuvant imatinib after surgery. Laparoscopic resection is a safe and feasible method in large and giant GISTs with oncologic and long-term outcomes comparable to open surgery, and with better short-term outcomes.
\end{abstract}

Key words: gastrointestinal stromal tumor, total gastrectomy, giant, case report.

\section{Introduction}

Gastrointestinal stromal tumors (GISTs) include $80 \%$ of gastrointestinal $(\mathrm{Gl})$ mesenchymal tumors and originate from interstitial Cajal cells that play the role of an intestinal pacemaker [1-4]. These tumors constitute $0.1-3 \%$ of $\mathrm{Gl}$ malignancies and the stomach is the most common site for them [2, 5]. The GISTs can present at any age, but are most common in middle age, with a median age of 65 years $[6,7]$. The GISTs are usually solitary without any invasion to adjacent organs, but can be multi-lobar and can show extra- or intra-luminary growth or a combination of these types [3, 8, 9]. All GISTs larg- er than $2 \mathrm{~cm}$ or any increase in tumor size, also some features such as bleeding, necrosis, cystic changes, ulceration, irregularity of the tumor margins, tumoral echogenic heterogeneity in Gl endoscopy or endosonography that can be signs of malignancy, are indications for surgery $[10,11]$.

Surgical resection with clear margins is the cardinal and potentially curative treatment of GISTs, with the aim of minimal morbidity and optimal organ function preservation $[1,2,11,12]$. Because of hematogen spreading of these mesenchymal tumors, there is no need for classic lymph node dissection during resection, unless there are enlarged lymph nodes during surgery, which are suspicious for metastasis, so in 
most conditions, simple resections such as wedge resection are performed, though based on size and location of the tumor, subtotal or total gastrectomy may need to be performed $[6,10,12,13]$.

Today, laparoscopic resection of GISTs, especially for tumors smaller than $5 \mathrm{~cm}$, in many guidelines, such as those of the National Comprehensive Cancer Network (NCCN), is recommended as standard treatment $[9,12,14,15]$. Recent studies show the safety and feasibility of laparoscopic resection in large $(>5 \mathrm{~cm})$ and giant $(>10 \mathrm{~cm})$ GISTs with oncologic results comparable to those of open surgery $[4,15-17]$.

The presentation of GISTs is related to the size and location of the tumor, and the most common features are gastrointestinal bleeding (GIB), abdominal pain, nausea, vomiting, weight loss, and palpable mass $[8,12,16,18]$. The GIB is usually intraluminal and chronic and massive GIB is a rare presentation [19]. Multi-slice computed tomography scan (MSCT) can show a diagnostic large and heterogeneous mass with low attenuation area of bleeding, necrosis or cystic degeneration [16].

\section{Case report}

A 52-year-old woman with a body mass index of 22 was admitted to the emergency department due to acute massive GIB. She had undergone gastroscopy for abdominal pain, weight loss and chronic anemia 1 month before admission. In her endoscopy, a large ulcerative lesion in the cardia and lesser curvature had been seen, the diagnosis of which in biopsy was GIST. In her immunohistochemistry, CD117 and CD34 were positive.

Metastasis workup with chest computed tomography (CT) and abdominopelvic CT had been performed and there was no evidence of metastasis. In the CT scan, she had a large and lobulated mass in the cardia and proximal part of the stomach (Photo 1).

Imatinib $600 \mathrm{mg} / \mathrm{d}$ had been started 3 days before admission, but after massive GIB, the patient presented to the emergency department. She did not use other drugs and had no addiction or family history for GIST or other GI cancers. The patient underwent surgical and gastroenterology consultation. The gastroenterologist recommended urgent surgery after initial resuscitation and blood transfusion.

The patient had blood pressure (BP): 90/60, PR: 120, RR: 18 and T: 37.2 oral. Her hemoglobin ( $\mathrm{Hb}$ ) was $7 \mathrm{~g} / \mathrm{dl}$.
Resuscitation was initiated and 6 units of packed cells were transfused, and the patient was prepared for surgery after stabilization with HB: $9.5 \mathrm{~g} / \mathrm{dl}$. The patient was operated on under general anesthesia in the reverse Trendelenburg and $\mathrm{V}$ position, and the surgeon stood between her legs. After $\mathrm{CO}_{2}$ insufflation, 5 trocars were inserted: one $12 \mathrm{~mm}$ trocar above the umbilicus in the midline for the camera, one $12 \mathrm{~mm}$ trocar in the left upper quadrant, and three $5 \mathrm{~mm}$ trocars in the right upper quadrant, left subcostal, and subxyphoid for the right working hand, left working hand, assistant and liver retractor respectively. After inspection of the abdominal cavity, as expected, a large and lobulated mass was found in the proximal part of the stomach that involved the lesser curvature. For identification of the tumor margin, gentle palpation of the stomach with a grasper was performed, and it was found that a part of tumor was intraluminal. Then we explored all of the abdominal cavity for any metastasis, which was negative. Due to the location and large size of the tumor (as shown in Photo 1), we decided to perform total gastrectomy, instead of proximal gastrectomy, which is more acceptable due to better physiologic outcomes and fewer complications [20]. The distal esophagus and proximal part of the duodenum were divided with a linear endostapler, and then esophagojejunostomy with a circular endostapler was performed. The next step was jejunojejunostomy performed with an endostapler (Photos $2 \mathrm{~A}-\mathrm{E})$.

Any enlarged lymph node was dissected during gastrectomy, due to the necessity of resection of enlarged lymph nodes, which may be a rare sign of metastasis in GISTs based on Asian Consensus Guidelines [10], and we avoided extensive lymph node dissection (LND) due to the nature of GISTs.

The sample was extracted in a large endobag through a Pfannenstiel incision. On the second day after the operation, a liquid regimen was initiated for the patient, and she was discharged on the fourth day after the operation. One week after operation, the sutures were removed. In the permanent pathologic report, a huge fragile lobulated creamy soft mass measuring $13 \times 8 \times 6 \mathrm{~cm}$ with hemorrhagic foci, at $0.5 \mathrm{~cm}$ and $18 \mathrm{~cm}$ distances from the proximal and distal margin, respectively, was recorded. The mass was mainly located in the submucosal layer with extension to the muscularis propria. All separated 18 lymph nodes were free of tumor.

The mitotic rate was 30/50 HPF, and in IHC CD34, CD 117, Ki-67 and S100 were positive, which 
suggested a grade 2 spindle cell type GIST, in the high-risk category. Based on these findings, imatinib $400 \mathrm{mg} /$ day was initiated for the patient, and she was advised that it must be continued for 3 years.

\section{Discussion}

Although there are still debates about the laparoscopic resection of large GISTs exceeding $5 \mathrm{~cm}$, there are some new studies that confirm the safety and feasibility of this method $[4,5,9,15,17]$.

Based on the size and site of GISTs, the surgical approach may be identified as wedge resection, sleeve, partial or total gastrectomy [21]. A very important issue in laparoscopic resection of GISTs is prevention of rupture and spillage of the tumor, which may lead to GIST implantation and high locoregional recurrence such as peritoneal sarcomatosis $[5,6,12]$. Oncologic outcomes are also observed to be the same in open resection (OR) and laparoscopic resection (LR) of small and large GISTs, even in the long term $[2,5,9,10,20]$.

The advantages of LR are less bleeding, less pain, faster return of bowel function, earlier initiation of oral regimen, shorter hospital stay, faster recovery, smaller incisions and better view of identifying the progression of the tumor to the liver or peritoneum $[2,5,9,14,17]$. In IHC CD117 (C-kit) is positive in $95-100 \%$ of GISTs, and CD34 is positive in some cases. Sometimes S100, which is a neuro-
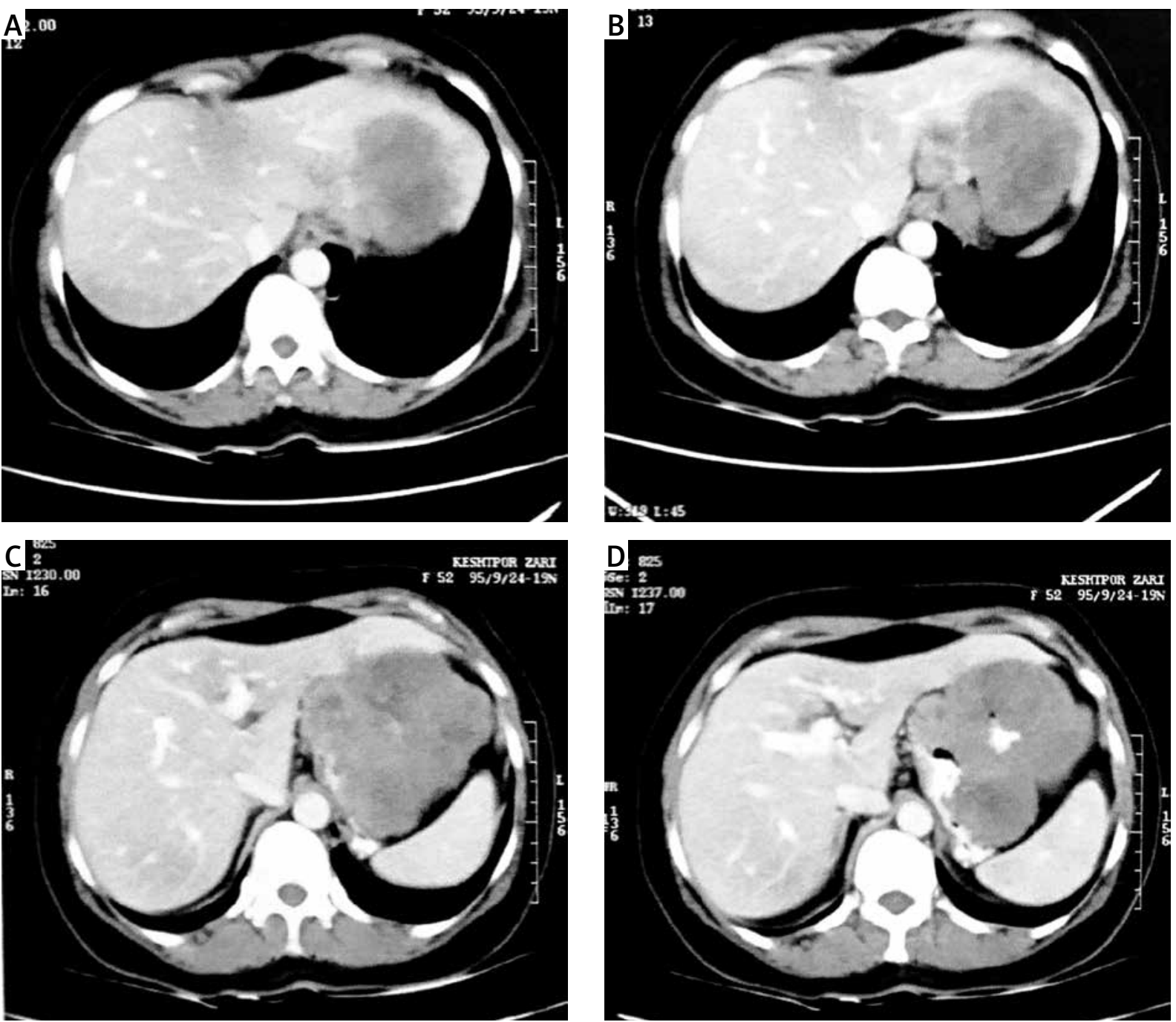

Photo 1. Abdominal computed tomography (CT) scan, showing a large lobulated mass in the cardia and proximal part of the stomach, including the lesser curvature 

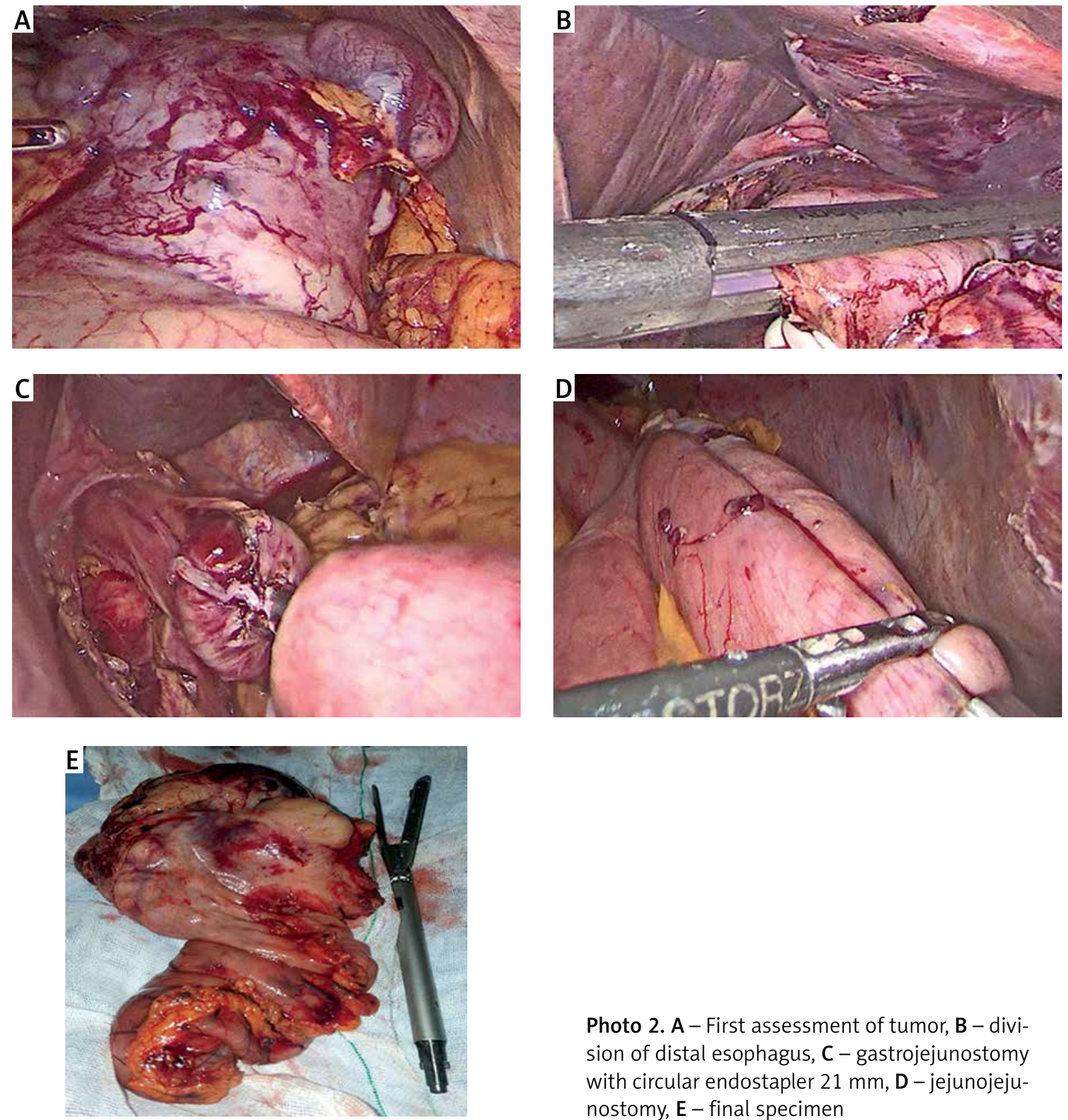

genic marker and is secreted in the Auerbach neural plexus and interstitial Cajal cells, is positive, which can show the neural differentiation process in GISTs [22-25].

Studies confirmed that the presence of CD34 and S100 has no effect on prognosis or survival of GISTs [25].

In fact, the factors that lead to high risk GISTs and high recurrence include tumor size $>10 \mathrm{~cm}$, mitotic rate $>10 / 50 \mathrm{HPF}$, rupture of tumor, non-gastric

Photo 2. A - First assessment of tumor, B - division of distal esophagus, $\mathbf{C}$ - gastrojejunostomy with circular endostapler $21 \mathrm{~mm}, \mathrm{D}$ - jejunojejunostomy, $\mathrm{E}$ - final specimen

site and adjacent organ involvement $[4,11,18]$. In high-risk GISTs, as in the case of our patient, adjuvant imatinib $400 \mathrm{mg} /$ day must be initiated and continued for 3 years $[10,11]$. Guidelines also recommend neoadjuvant imatinib 400-600 mg/day to shrink large GISTs for less resection and organ function preservation [10, 11]. Our patient had been receiving neoadjuvant imatinib, but she had GIB after 3 days and so underwent laparoscopic surgical resection. 


\section{Conclusions}

It can be concluded that laparoscopic resection is a safe and feasible method in large and giant GISTs with oncologic outcomes comparable to the open method and better short-term outcomes with significant advantages, but must be done with some principles such as prevention of tumoral rupture during surgery, removal of the specimen in a plastic bag, adjuvant imatinib and close follow-up.

\section{Acknowledgments}

We gratefully appreciate the patient and her family for their cooperation in this manuscript.

\section{Conflict of interest}

The authors declare no conflict of interest.

\section{References}

1. Navarrete A, Momblán D, Almenara R, Lacy A. Giant gastric gastrointestinal stromal tumor (GIST). J Gastrointest Surg 2017; 21: 202-4.

2. Khoo CY, Goh BKP, Eng AKH, et al. Laparoscopic wedge resection for suspected large $(\geq 5 \mathrm{~cm})$ gastric gastrointestinal stromal tumors. Surg Endosc 2017; 31: 2271-9.

3. Kim SH, Lee MS, Cho BS, et al. Gastrointestinal stromal tumor of the stomach presenting as multilobular with diffuse calcifications. J Gastric Cancer 2016; 16: 58-62.

4. Milone M, Elmore U, Musella M, et al. Safety and efficacy of laparoscopic wedge gastrectomy for large gastrointestinal stromal tumors. Eur J Surg Oncol 2017; 43: 796-800.

5. Severino BU, Fuks D, Lainas P, et al. Large gastrointestinal stromal tumours of the stomach: is laparoscopy reasonable? I Minim Access Surg 2016; 12: 148-53.

6. Nishida T, Goto O, Raut CP, Yahagi N. Diagnostic and treatment strategy for small gastrointestinal stromal tumors. Cancer 2016; 122: 3110-8.

7. Stotz M, Liegl-Atzwanger B, Posch F, et al. Blood-based biomarkers are associated with disease recurrence and survival in gastrointestinal stroma tumor patients after surgical resection. PloS One 2016; 11: e0159448.

8. Maeda E, Fujiwara M, Suto $\mathrm{H}$, et al. Multiple gastric gastrointestinal stromal tumors treated by laparoscopic-endoscopic cooperative surgery: a case report. Asian J Endoscop Surg 2016; 9: 336-9.

9. MacArthur KM, Baumann BC, Nicholl MB. Laparoscopic versus open resection for gastrointestinal stromal tumors (GISTs). J Gastroint Cancer 2017; 48: 20-4.

10. Koo DH, Ryu MH, Kim KM, et al. Asian consensus guidelines for the diagnosis and management of gastrointestinal stromal tumor. Cancer Res Treat 2016; 48: 1155-66.

11. Von Mehren M, Benjamin RS, Bui MM, et al. Soft tissue sarcoma, version 2.2012 featured updates to the NCCN guidelines. J Natl Compr Cancer Netw 2012; 10: 951-60.
12. Smolarek S, Pomeroy E, Kinnarney F, Arumugasamy M. Laparoscopic resection of large gastric gastrointestinal stromal tumours. Videosurgery Miniinv 2016; 11: 31-7.

13. Okumura S, Kanaya S, Hosogi $\mathrm{H}$, et al. Our experience with laparoscopic partial gastrectomy by the 'lift-and-cut method' for gastric gastrointestinal stromal tumor with maximal preservation of the remnant stomach. Surg Endosc 2016 in press.

14. Hu J, Or BH, Hu K, Wang ML. Comparison of the post-operative outcomes and survival of laparoscopic versus open resections for gastric gastrointestinal stromal tumors: a multi-center prospective cohort study. Int J Surg 2016; 33: 65-71.

15. Demetri GD, von Mehren M, Antonescu CR, et al. NCCN Task Force report: update on the management of patients with gastrointestinal stromal tumors. J Natl Compr Cancer Netw 2010; 8 (Suppl. 2): S1-41.

16. Wang Y, Peng J, Huang J. Giant and high-risk gastrointestinal stromal tumor in the abdomino-pelvic cavity: a case report. Oncol Letters 2016; 11: 2035-8.

17. Ismael H, Ragoza Y, Caccitolo J, Cox S. Optimal management of GIST tumors located near the gastroesophageal junction: case report and review of the literature. Int I Surg Case Rep 2016; 25: 91-6.

18. Bard V, Menasherov N, Morgenstern S, et al. Gastrointestinal stromal tumor of stomach: a gentle enemy of the surgeon. Our experience in confronting the disease. Surg Laparosc Endosc Percutan Tech 2016; 26: 406-9.

19. Srinivasan PH, Maliekal JI, Reddy CS, Suda S. GIST Jinx - a rare case of acute massive life threatening bleed from a jejunal gastro-intestinal stromal tumour. J Clin Diagn Res 2016; 10: PJ03-4.

20. Chen QF, Huang CM, Lin M, et al. Short-and long-term outcomes of laparoscopic versus open resection for gastric gastrointestinal stromal tumors: a propensity score-matching analysis. Medicine 2016; 95: e3135.

21. Ong E, Abrams Al, Lee E, Jones C. Single-incision sleeve gastrectomy for successful treatment of a gastrointestinal stromal tumor. JSLS 2013; 17: 471-5.

22. Salari M, Ahadi M, Hoseini SM, et al. Gastrointestinal stromal tumors in Northeastern Iran: 46 cases during 2003-2012. Middle East J Dig Dis 2015; 7: 161-5.

23. Terad T. Histopathological study using computer database of 10000 consecutive gastric specimens: (2) malignant lesions. Gastroenterol Rep 2016; 4: 54-8.

24. Terada T. Smooth muscles and stem cells of embryonic guts express KIT, PDGFRRA, CD34 and many other stem cell antigens: suggestion that GIST arise from smooth muscles and gut stem cells. Int J Clin Exp Pathol 2013; 6: 1038-45.

25. Liu Q, Wang Y, Kong L, Kan Y. Study on clinicopathological features of gastrointestinal stromal tumor and relevant prognostic factors. Cell Biochem Biophys 2015; 73: 743-7.

Received: 8.03.2017, accepted: 26.04.2017. 